Supporting Information:

\title{
A Comparative Study on Different Carbon Nanotube Materials in Terms of Transparent Conductive Coatings
}

\author{
Zhongrui Li, ${ }^{1 *}$ Hom R. Kandel, ${ }^{2}$ Enkeleda Dervishi, ${ }^{1}$ Viney Saini, ${ }^{1}$ Yang Xu,,${ }^{1}$ Alexandru
} R. Biris, ${ }^{3}$ Dan Lupu, ${ }^{3}$ Gregory J. Salamo ${ }^{4}$, Alexandru S. Biris ${ }^{1}$

1. Nanotechnology Center, University of Arkansas at Little Rock, Arkansas 72204

2. Department of Physics and Astronomy, University of Arkansas at Little Rock, Arkansas 72204

3. National Institute for Research and Development of Isotopic and Molecular Technologies, P.O. Box 700, R-400293 Cluj-Napoca, Romania

4. Physics Department, University of Arkansas, Fayetteville, Arkansas, 72701 


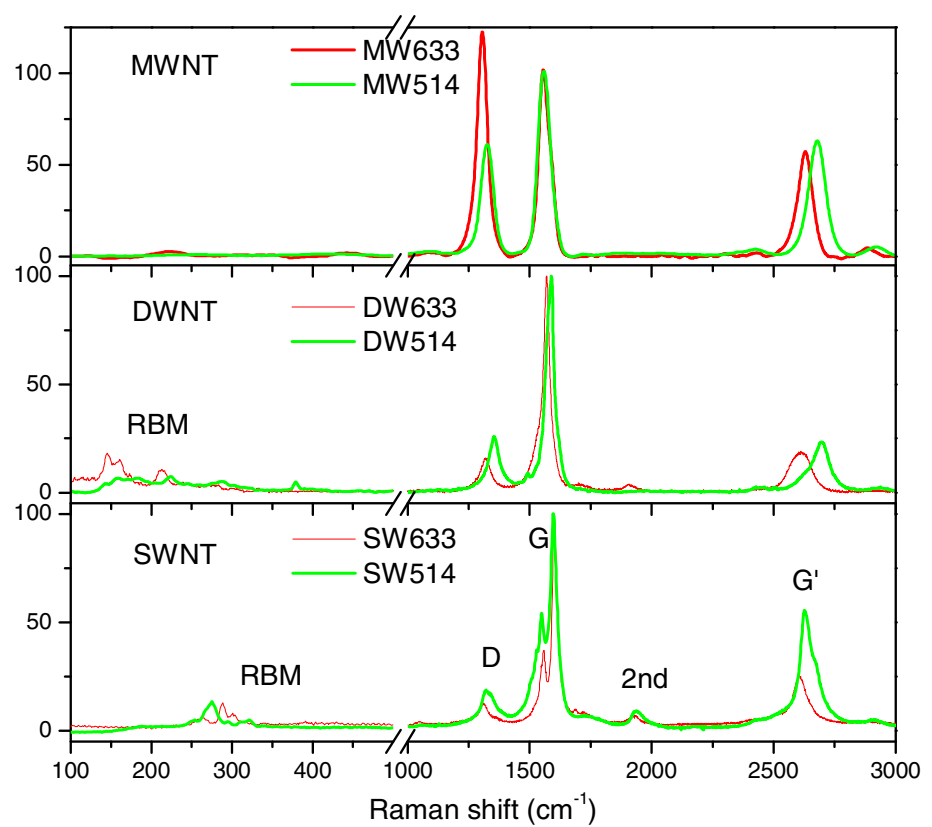

Figure S1. Raman scattering from the SWNT, DWNT and MWNT under different laser excitations (wavelength $632.8 \mathrm{~nm}$, and $514.0 \mathrm{~nm}$ ). 


\section{\#2. The cross sectional SEM images of SWNT on membrane filter}

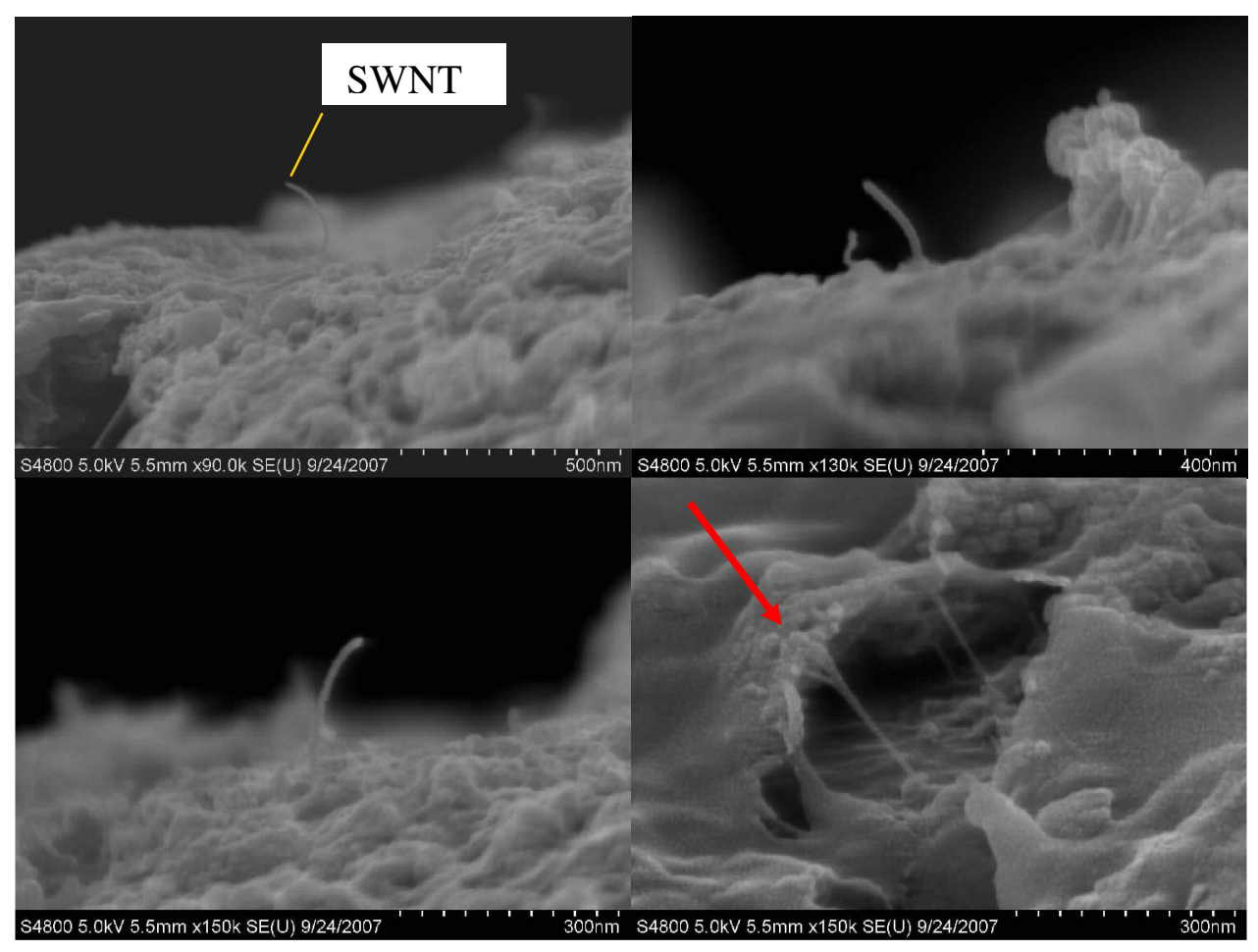

Figure S2. The cross sectional SEM images of the SWNTs/polycarbonate membrane filter. This sample was prepared to mimic the high transparent SWNT film by vacuum filtration of much diluted SWNT/sodium cholate solution. As seen in the above SEM images, the SWNTs were vertically aligned on the porous membrane by the flow in the vacuum filtration process. Some short tubes are also vertically trapped inside the pores of the membrane, as seen in the image at the right bottom. The red arrow marks the flow direction. 


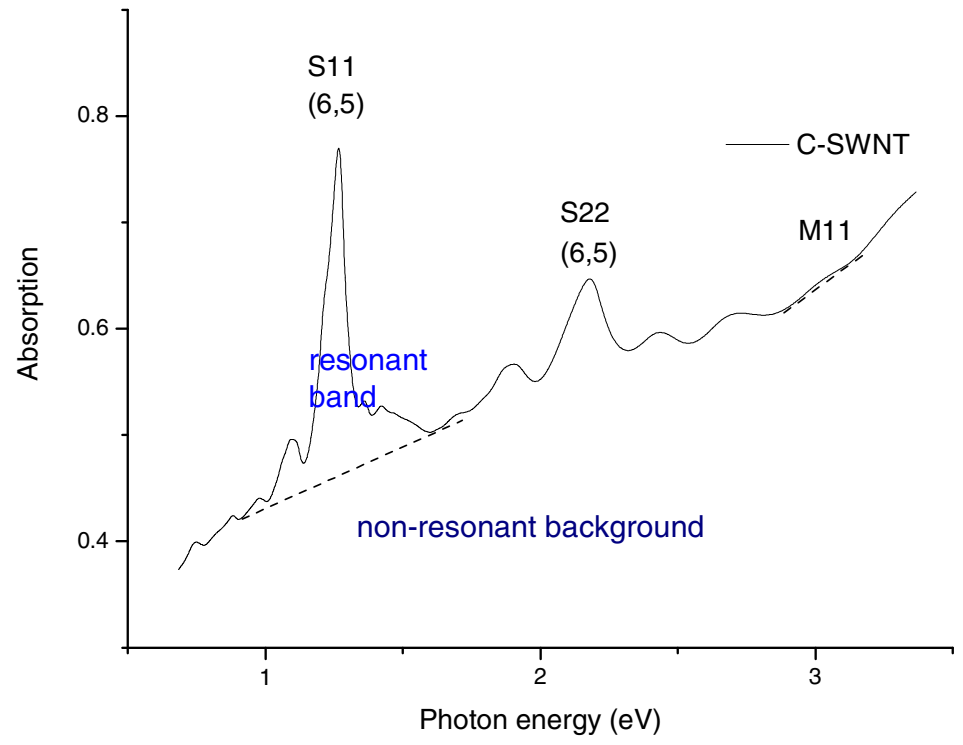

Figure S3. The semiconducting to metallic tubes ratio can be estimated from the optical absorption for the C-SWNT sample. 


\section{\#3: The quality of MWNT materials synthesized by two different methods: EF-CVD vs. RF-CVD.}

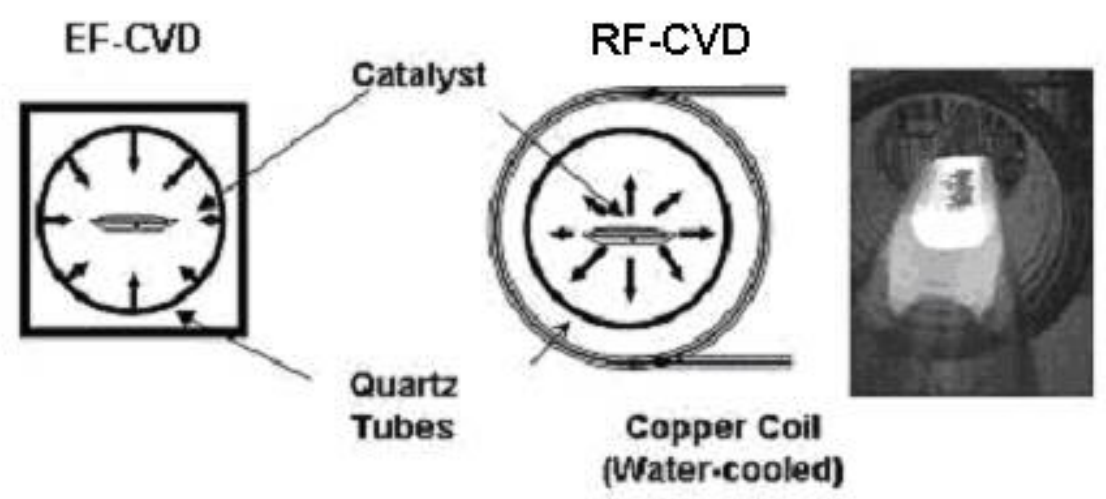

Figure S4. Schematic diagrams display both the resistive external furnace (EF) heating and inductive (RF) heating processes. The right picture shows the glowing susceptor inside the RF induction coil during the synthesis of multi-wall carbon nanotubes.

Catalyst preparation and MWNT synthesis:

EF-MWNT and RF-MWNTs were produced from the pyrolysis of acetylene on the same type of catalyst, $\mathrm{Fe}-\mathrm{Co} / \mathrm{CaCO}_{3}$ with $\mathrm{Fe} 2.5 \mathrm{wt} \%$ and $\mathrm{Co} 2.5 \mathrm{wt} \%$, see [Chem. Mater. 2007, 19, 179-184]. The experimental conditions for the synthesis of the EF-MWNTs was described in [Chem. Mater. 2007, 19, 179-184].

In the synthesis of the RF-MWNTs, around $200 \mathrm{mg}$ of the catalyst was uniformly spread into a thin layer under $\mathrm{N}_{2}$ flow at $300 \mathrm{ml} / \mathrm{min}$ on a graphite susceptor and placed at the center of a quartz tube positioned horizontally inside an inductive furnace. After purging the system with nitrogen as carrier gas $(300 \mathrm{ml} / \mathrm{min})$ for 10 minutes, radio frequency (RF) heating at $350 \mathrm{KHz}$ was applied to the graphite susceptor that contains the catalyst. After the temperature reached 720 ${ }^{\circ} \mathrm{C}$, and acetylene was introduced at $3.3 \mathrm{ml} / \mathrm{min}$ for about 30 minutes. Neither nanotubes nor any other types of carbon byproducts were found in the experiments performed only with a graphite susceptor without catalyst. The as-produced CNTs were purified in one easy step using diluted hydrochloric acid solution $(1: 1 \mathrm{v} / \mathrm{v})$ for 30 minutes, followed by distilled water wash and drying at $120{ }^{\circ} \mathrm{C}$ overnight. 

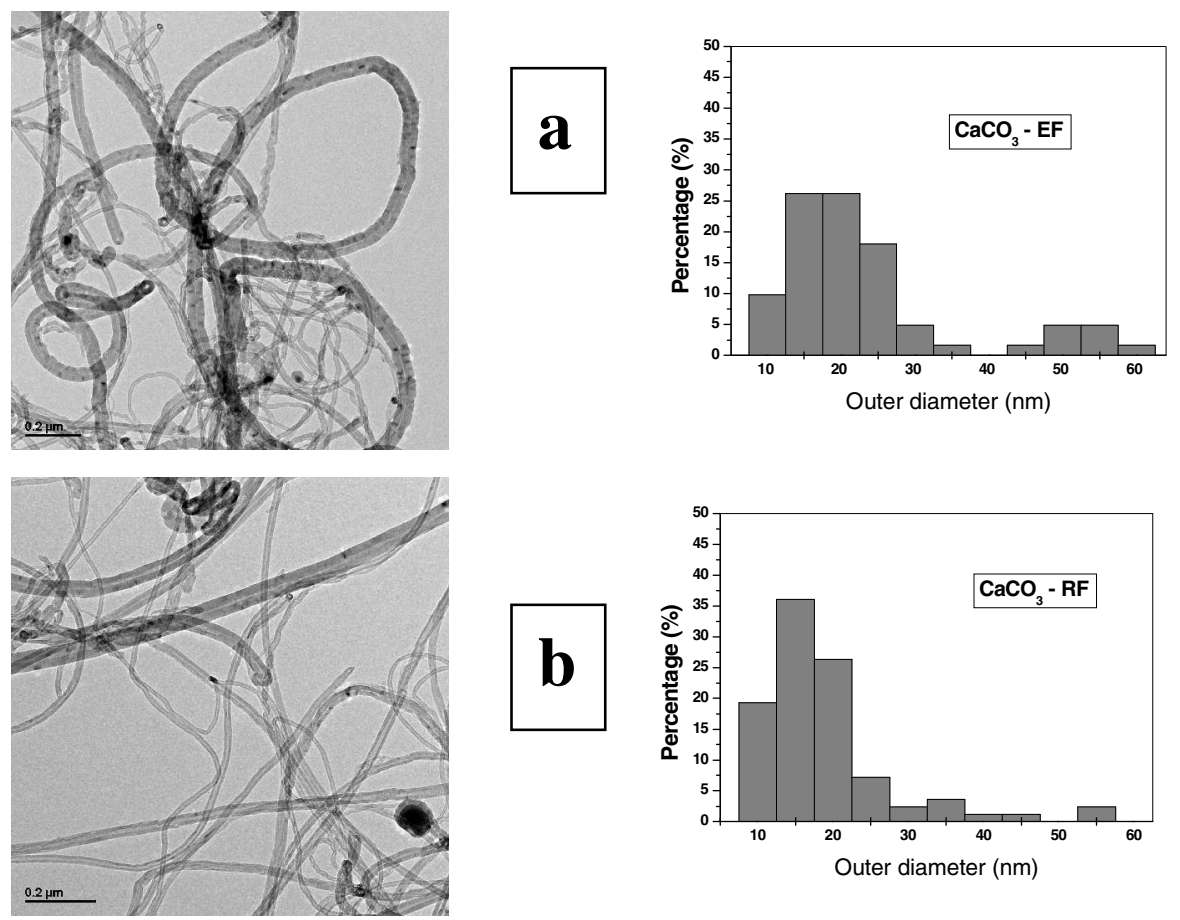

Figure S5. The low-magnification TEM images and statistical analysis illustrating the diameter distributions of the nanotubes synthesized by both EF- and RF-CVD methods. The EF-CVD grown nanotubes have outer diameter between 8 and $65 \mathrm{~nm}$. The RF-grown nanotubes have outer diameter between 5 and $55 \mathrm{~nm}$. 95\% of nanotubes grown by RF-CVD have outer diameters between 5 and $35 \mathrm{~nm}$. The RF-MWNTs exhibit 3 to 7 carbon layers with outer/inner diameter (OD/ID) ratios ranging between 2.0 and 2.5. The EF-MWNTs show OD/ID ratios ranging from 4.5 to 6.0 with a much larger number of graphitic walls (over 20). 


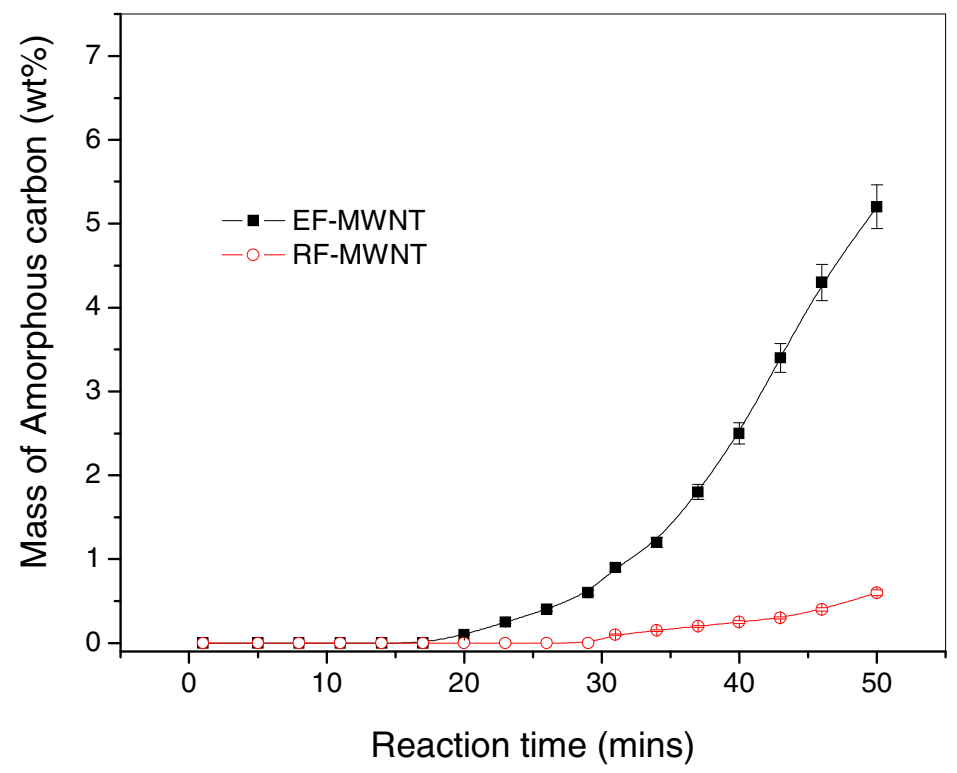

Figure S6. The amount of amorphous carbon generated as a function of reaction time for two different heating methods. Amount of amorphous carbon was studied using TGA by measuring mass loss in temperature interval of 200 to $480{ }^{\circ} \mathrm{C}$. The amount of amorphous carbon formed by EF-CVD was about $5 \mathrm{wt} \%$ while that by RF-CVD was less than 0.6 wt $\%$ after 50 minutes of reaction.

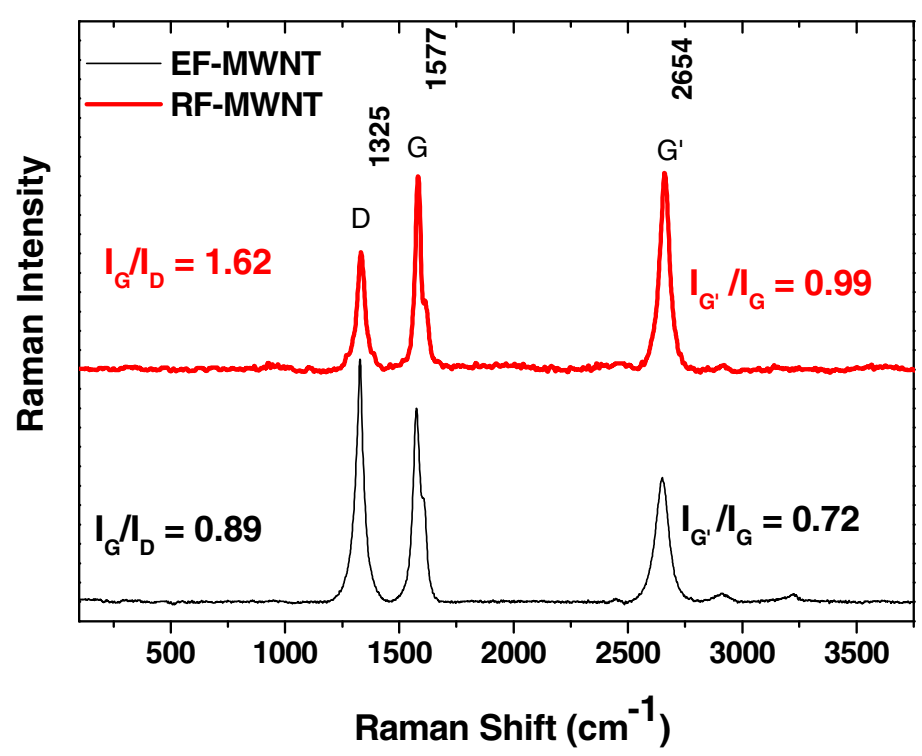

Figure S7. The Raman scattering spectra from the EF- and RF-MWNTs. The higher $I_{G} / I_{D}$ and $\mathrm{I}_{\mathrm{G}}, \mathrm{I}_{\mathrm{G}}$ values of the RF-MWNTs indicate higher quality. 